\title{
VARIAÇÕES ANUAIS NA PRODUÇÃO DE FRUTOS E SEMENTES DE CASTANHEIRA-DO-BRASIL (Bertholletia excelsa Bonpl., Lecythidaceae) EM FLORESTAS NATIVAS DE RORAIMA ${ }^{1}$
}

\author{
Helio Tonini² e Cássia Ângelo Pedrozo ${ }^{3}$
}

\begin{abstract}
RESUMO - Este trabalho foi desenvolvido com o objetivo de estudar as variações anuais na produção de frutos e sementes entre populações e indivíduos em castanhais nativos de Roraima. Os dados foram obtidos em cinco parcelas permanentes de 300 m x 300 m instaladas nos Municípios de Caracaraí e São João da Baliza. Nas parcelas permanentes, todas as castanheiras com diâmetro à altura do peito (DAP) superior ou igual a $10 \mathrm{~cm}$ foram identificadas, medidas e numeradas através de placas de alumínio presas aos fustes por pregos. Durante o período de queda dos frutos (março a julho), as árvores foram visitadas em duas ocasiões no meio e no final desse período, e os frutos contados, abertos e pesados com balança de gancho digital com precisão de 50 g. A produção de frutos e sementes varia entre populações e indivíduos e a maioria não produz frutos todos os anos. No ano de maior produção, um castanhal chega a produzir 52 vezes mais do que em anos de baixa. Nos locais estudados, a produção total concentra-se em um pequeno número de indivíduos com um ano de pico de produção (mast-year).
\end{abstract}

Palavras-chave: Amazônia; Monitoramento; Produto florestal não madeireiro.

\section{CHANGES IN ANNUAL PRODUCTION OF FRUITS AND SEEDS OF BRAZILIAN NUTS TREES (Bertholletia excelsa Bonpl., Lecythidaceae) IN NATIVE FORESTS OF RORAIMA STATE, BRAZIL}

\begin{abstract}
The objective of the present study was to evaluate annual production variations between populations and individuals in native Brazilian nut groves of Roraima. Data were obtained from five permanent plots of $300 \mathrm{~m} \times 300 \mathrm{~m}$ installed in the municipalities of Caracaraí and São João da Baliza. Every tree presenting the diameter at breast height $(D B H)$ greater than or equal to $10 \mathrm{~cm}$ were measured and identified using aluminum number labels attached to the stem by nails. During the falling of fruits (March-July) all the trees were visited on two occasions: in the middle and at the end of this period. The fruits of each tree were counted, and seeds were weighed using a digital balance scale accurate to $50 \mathrm{~g}$. The fruit and seed production varied among populations and individuals, and the majority did not produce fruits every year. In a high production year, the yield is up to fifty-two times greater than in the low production years. The total production was concentrated on a small number of trees with one year of peak production (mast-year).
\end{abstract}

Keywords: Amazon; Monitoring; Non-timber forest products.

\section{INTRODUÇÃO}

As florestas tropicais são ricas em espécies animais e vegetais, e a exploração sustentável de produtos florestais não madeireiros é considerada uma das estratégias que melhor conservam essa biodiversidade. No entanto, o desenvolvimento e implementação desses sistemas de exploração ainda carecem de informações referentes à sustentabilidade biológica, econômica e social (BOOT, 1997).

Apesar do crescente interesse científico, as questões referentes à produtividade e ao impacto ecológico da extração de produtos florestais não

\footnotetext{
${ }^{1}$ Recebido em 20.12.2012 aceito para publicação em 06.11.2013.

${ }^{2}$ Empresa Brasileira de Pesquisa Agropecuária, Embrapa Agrossilvipastoril. E-mail: < helio.tonini@embrapa.br>.

${ }^{3}$ Empresa Brasileira de Pesquisa Agropecuária, EMBRAPA, Brasil. E-mail: <cassia.pedroso@embrapa.br>.
} 
madeireiros permanecem, ainda, sem resposta, mesmo para espécies de grande interesse comercial (PHILLIPS,1993), como a castanheira-do-brasil (Bertholletia excelsa Humb. \& Bonpl., Lecythidaceae).

Entre os produtos florestais não madeireiros da Amazônia, a semente da castanheira-do-brasil é o mais conhecido e solidamente estabelecido nos mercados doméstico e de exportação. Sua coleta é ainda realizada quase que exclusivamente em florestas tropicais primárias (PERES et al., 2003; SALOMÃO, 2009), o que a torna espécie-chave para o desenvolvimento econômicosocial das comunidades e para a manutenção dos benefícios diretos e indiretos da floresta.

Estudos realizados por Viana et al. (1998), Zuidema e Boot (2002), Wadt et al. (2005) e Tonini et al. (2008) determinaram a produção da castanheira-do-brasil em diferentes regiões da Amazônia; no entanto, somente Kainer et al. (2007) analisaram dados em uma série relativamente longa de anos de produção.

Para árvores de alto valor econômico, como a castanheira-do-brasil, um máximo nível de frutificação e uma mínima variação interanual podem ser fatores decisivos no sucesso comercial (KAINER et al., 2007) e, consequentemente, na valorização do recurso florestal. Além disso, árvores que apresentam elevada produtividade e repetitibilidade de produção ao longo dos anos são de grande interesse para a produção de mudas de maior qualidade genética.

Além da quantificação ao longo dos anos, a quantificação da produção por árvore, que varia entre diferentes populações, é fundamental para estimativa adequada da produtividade potencial em determinada área ou região (ZUIDEMA, 2003) e tem importância central na conservação e manejo da castanheira-do-brasil.

Nesse contexto, este trabalho foi desenvolvido com o objetivo de estudar as variações anuais e interanuais de produção de frutos e sementes entre populações e indivíduos em castanhais nativos de Roraima.

\section{MATERIAL E MÉTODOS}

\subsection{Características dos locais de coleta dos dados}

As áreas experimentais estão localizadas no Estado de Roraima, nos Municípios de Caracaraí e São João da Baliza (Figura 1), e consistem de cinco parcelas permanentes de 9 ha (300 x 300 m), instaladas pela Embrapa Roraima entre 2005 e 2008, com o objetivo de estudar a dinâmica e produção de frutos e sementes em populações nativas de castanheira-do-brasil.

No Município de Caracaraí, foram instaladas quatro parcelas permanentes em castanhais localizados em duas regiões distantes $50 \mathrm{~km}$. Na região do Projeto de Assentamento do Itã (ITA), uma parcela permanente foi instalada em 2006 e outra em 2007, em um fragmento florestal de 400 ha, na Fazenda Pau-Rainha ( $01^{\circ} 48^{\prime}$ $58,0^{\prime \prime}$ Norte e $61^{\circ} 07^{\prime} 41,0^{\prime \prime}$ Oeste). Outras duas parcelas foram instaladas em 2008, em uma área de Plano de Manejo Florestal Sustentável, na Fazenda Mundo Novo, com uma área de 17.000 ha na região do Projeto de Assentamento do Cujubim - CUJ (01 ${ }^{\circ}$ 51' 02,6”' Norte e $61^{\circ} 00^{\prime} 30,6^{\prime \prime}$ ' Oeste). A quinta parcela permanente analisada foi instalada em 2006, na reserva legal de um lote de assentamento rural denominado Sítio Umuarama, em São João da Baliza - SJB (00 57’02" Norte e 5954’41" Oeste.

Em Caracaraí, o clima da região é classificado como Ami (tropical chuvoso, com pequeno período de seca). Em São João da Baliza, é do tipo Awi (tropical úmido com pequeno período de seca durante o ano) e Aji (tropical chuvoso sem estação seca). O período caracteristicamente seco está compreendido entre os meses de novembro e março (FEMACT, 1993). Segundo dados obtidos no INMET, no período de estudo a precipitação na região variou entre $1.661 \mathrm{~mm}$ (2009) e 2.623,2 mm (2006), com uma média de 2.293,06 mm.

O solo predominante na Fazenda Pau-Rainha é o Argissolo, com colorações entre Amarelo e VermelhoAmarelo, conforme a classificação realizada pela equipe no local. Já o solo predominante na Fazenda Mundo Novo, Argissolo Vermelho-Amarelo, com presença de afloramentos rochosos em algumas áreas, segundo informações fornecidas pela Madeireira Vale Verde, proprietária da área, e confirmadas pela equipe em campo. No Sítio Umuarama, a classificação não foi realizada, porém predominam na região os solos ArgissoloAmarelo Distrófico e LatossoloAmarelo Distrófico (SEPLAN, 2010).

\subsection{Coleta e análise dos dados}

Os dados de produção foram coletados de 2006 a 2012 em SJB e ITA e de 2009 a 2012 em CUJ. Durante esse período, foram monitoradas 385 árvores, sendo 34 em SJB (3,8 árvores ha-1), 238 em ITA (13,2 árvores ha $\left.{ }^{-1}\right)$ e 113 em CUJ (6,3 árvores ha-1). 


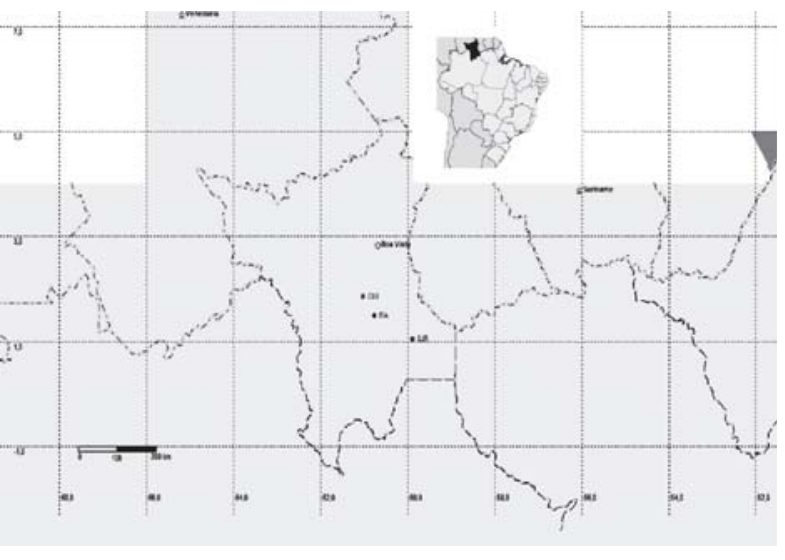

Figura 1 - Localização geográfica do Estado de Roraima e dos três castanhais estudados (SJB, ITA, CUJ).

Figure 1 - Geografic Location of the state of Roraima and the tree brazil nut groves studied (SJB, ITA, CUJ).

Nas parcelas permanentes, todas as castanheiras com diâmetro à altura do peito (DAP) superior ou igual a $10 \mathrm{~cm}$ foram identificadas, medidas e numeradas através de placas de alumínio pregadas nos fustes. Durante o período de queda dos frutos (março a julho), todas as árvores foram visitadas em duas ocasiões, sendo a primeira em maio e a segunda em julho, representando o meio e o final desse período. Em cada árvore, todos os frutos foram contados, abertos e pesados, utilizando uma balança de gancho digital com precisão de 50 g.

Os pressupostos da homogeneidade de variância e normalidade dos dados de produção foram verificados pelos testes de Levene e Kolmogorov-Smirnov. Nenhuma transformação adotada (logaritmo neperiano, decimal e raiz quadrada) atendeu aos pré-requisitos para a aplicação da estatística paramétrica, uma vez que os dados não apresentaram distribuição normal e homogeneidade de variância.

Na análise intraparcelar da produção entre os anos, optou-se pela aplicação da estatística não paramétrica para medições repetidas no tempo (grupos relacionados), com a utilização da análise de variância de Friedman e posterior comparação de médias, aplicando o teste descrito em Siegel e Castellan (1988), em que a diferença modular entre as médias dos rankings é comparada a um valor crítico calculado da seguinte forma:

$$
\left|\bar{R}_{u}-\bar{R}_{v}\right| \geq z_{\partial} / k(k-1) \sqrt{\frac{k(k+1)}{6 N}}
$$

em que $\mathrm{Ru}=$ média do ranking $\mathrm{u} ; \mathrm{Rv}=$ média do ranking v; zá = valor tabelado de Z para um menor valor de $\alpha /(k(k-1)) ; \mathrm{k}=$ número de grupos comparados; e $\mathrm{N}$ = número de observações.

Para análise da produção entre parcelas (dados independentes), adotaram-se os testes de Kruskal-Wallis e Wilcoxon, aplicando a Correção de Bonferroni no caso de mais de uma comparação de médias.

Para quantificar a variação anual na produção de sementes e frutos, utilizou-se o coeficiente de variação, conforme Kelly e Sork ( 2002) e Snook et al. (2005). A variabilidade da população no número de frutos produzidos foi medida pelo coeficiente de variação médio $\left(\mathrm{CV}_{\mathrm{p}}\right)$ resultante da variabilidade individual por ano/local. A variabilidade individual $\left(\mathrm{CV}_{\mathrm{i}}\right)$ foi obtida pelo cálculo da média do coeficiente de variação do número de frutos produzidos por árvore em todos os anos.

Para representar espacialmente a produção individual por classes de tamanho e produtividade, todas as árvores foram georreferenciadas e tiveram as coordenadas cartesianas registradas. As árvores foram classificadas em três classes diamétricas (DAP): pequenas: $\mathrm{d} \leq 50 \mathrm{~cm}$; médias (entre 50 e $100 \mathrm{~cm}$ ) e grandes (maior que $100 \mathrm{~cm}$ ); e de produtividade: (nula; baixa = média de produção igual ou abaixo da populacional; e alta = média de produção acima da populacional).

\section{RESULTADOS}

\subsection{Variações na produção de frutos e sementes entre locais e anos}

A produção de frutos e sementes variou significativamente entre locais e dentro de um mesmo local, com maior produção em 2012 e menor em 2011. No ano de maior produção, os castanhais produziram de 8 a 52 vezes mais do que no ano de menor produção.

A produção média por árvore diferiu entre locais nos anos de observação. Na comparação SJB e ITA, as diferenças foram significativas apenas no ano de 2008 ( $U$ = 1094, p = 0,001). Na comparação entre os três locais, a partir de 2009 ocorreram diferenças significativas em todos os anos (2009: $\chi(2)=92,5$; $\mathrm{p}=0,001 ; 2010: \chi(2)=44,4 ; \mathrm{p}=0,001 ; 2011: \chi(2)=$ $98,9 ; \mathrm{p}=0,001 ; 2012: \chi(2)=33,6 ; \mathrm{p}=0,001)$, sendo a média sempre maior na região do Cujubim. 
O número médio de frutos produzidos por árvore $\mathrm{ano}^{-1}$, desconsiderando as árvores com DAP $\leq 50 \mathrm{~cm}$, consideradas imaturas (WADT et al., 2005), variou por local, com valores médios entre 21 e 115 . O número máximo de frutos observados por árvore foi de 1.340 frutos. O peso médio de sementes por árvore e local variou entre 3,4 e 13,9 kg, com um máximo de 173,8 kg arvore $^{-1}$.

A produção anual em peso de sementes e número de frutos diferiu significativamente entre os anos, em todos os locais estudados: $\mathrm{SJB}(\chi(6)=21,85 ; \mathrm{p}=0,001$; $\chi(6)=23,56 ; \mathrm{p}=0,001 ; \operatorname{ITA}(\chi(6)=197,4 ; \mathrm{p}=0,001$; $\chi(6)=195,03 ; \mathrm{p}=0,001)$ e CUJ $(\chi(3)=139,5 ; \mathrm{p}=0,001$; $\chi(3)=145,8$; е $\mathrm{p}=0,001$ ).

A produção média das árvores em um mesmo local diferiu entre os anos. Em SJB, ocorreram diferenças significativas na média de produção das árvores apenas entre os anos 2011 e 2012 ( $Z=3,118$, p = 0,001). Em CUJ, as médias diferiram significativamente em todos os anos. Em ITA, a produção diferiu significativamente na maioria das comparações, sendo semelhantes apenas nos anos 2006 e 2009 ( $Z=657$; p = 0,511).

Em SJB, a produção média por árvore nos sete anos foi de 4,2 kg (variando de 2,2 a 10,5 kg) e 26 frutos (variando entre 12 e 64). Desconsiderando a produção das árvores imaturas, a média aumentou para 6 kg (variação de 2,9 a 14,2 kg) e 35 frutos (variação de 18 a 37). Os altos valores do desvio-padrão indicaram grandes variações de produção, mesmo entre árvores consideradas maduras.

Em ITA, a média por árvore foi de 3,4 kg (variando de 0,1 a 8,0 kg) e 21 frutos (variando de 2 a 68). Desconsiderando a produção das árvores imaturas, a média aumentou para 4, 2 kg (variação de 0,2 a 10,2 kg) e 27 frutos (variação de 2 a 68).

Em CUJ, a média por árvore foi a mais alta, com 13,2 kg (variação de 8,7 a 26 kg) e 115 frutos (variação de 21 a 216). Desconsiderando a produção das árvores imaturas, a média aumentou para 13,9 kg (variação de 2,7 a 27,7 kg) e 121 frutos (variação de 23 a 228).

As diferenças na produção média e na densidade dos castanhais refletiram-se na produção por hectare (Figura 2); porém, o padrão observado foi semelhante em todas as populações, que apresentaram um ano de alta produção (bem acima da média) em 2012, precedido por um ano de produção muito baixa (2011).
Em SJB, foi observada uma produção média de $16 \mathrm{~kg} \mathrm{ha}^{-1}$ de sementes, com densidade de árvores adultas de 2,5 árvores ha-1; em ITA, 34,8 kg ha-1 com 10,1 árvores adultas por hectare; e em CUJ, 166,18 $\mathrm{kg} \mathrm{ha}^{-1}$, com 6,3 árvores adultas por hectare. Esses resultados indicaram que a produção neste último local foi bastante superior, mesmo apresentando menor densidade de plantas adultas que ITA.

A variação populacional e individual no número de frutos produzidos no tempo, medida pelo coeficiente de variação, variou entre os locais estudados: SJB $\left(\mathrm{CV}_{\mathrm{p}}=0,27 ; \mathrm{CV}_{\mathrm{i}}=1,58\right)$, ITA $\left(\mathrm{CV}_{\mathrm{p}}=2,44 ; \mathrm{CV}_{\mathrm{i}}=1,67\right)$ e CUJ $\left(\mathrm{CV}_{\mathrm{p}}=1,43\right.$; e $\left.\mathrm{CV}_{\mathrm{i}}=1,11\right)$.

\subsection{Padrões espacial e temporal na produção de frutos}

Ao avaliar os padrões de frutificação das castanheiras maduras (DAP $\leq 50 \mathrm{~cm}$ ), observou-se que a maioria dos indivíduos não produziu frutos todos os anos. A proporção dos que produziram todos os anos variou entre populações, com $23,7 \%$ em SJB, 4,4\% em ITA e 47,8\% em CUJ.

Tomando como critério a média populacional, observou-se que os indivíduos considerados de melhor desempenho, ou seja, com produção acima dessa média, representaram uma fração considerável na população, com 50\% em SJB, 34\% em ITA e 34,5\% em CUJ. Em ITA, o padrão predominante foi o de produzir frutos em cinco dos sete anos (20,2\% das plantas), com a maioria dos indivíduos não produzindo em 2008 e 2011.

Em SJB, o padrão predominante foi o de produzir frutos em quatro dos sete anos (31,8\% das plantas); porém, não foi identificado um padrão comum entre essas árvores, uma vez que os anos de produção nula se alternaram entre os indivíduos. Curiosamente, cinco das sete árvores (71,4\% das plantas) não produziram em 2012, ano considerado de superprodução. Em CUJ, maior percentual de árvores (23\%) produziu em três dos quatro anos.

A construção de um ranking de produção permitiu observar que, entre as árvores mais produtivas em $\mathrm{SJB}$, apenas uma se manteve entre as 19 mais produtivas durante todos os sete anos. Em ITA e CUJ, isso não ocorreu, havendo alternância entre os indivíduos com maiores produções entre os anos. 

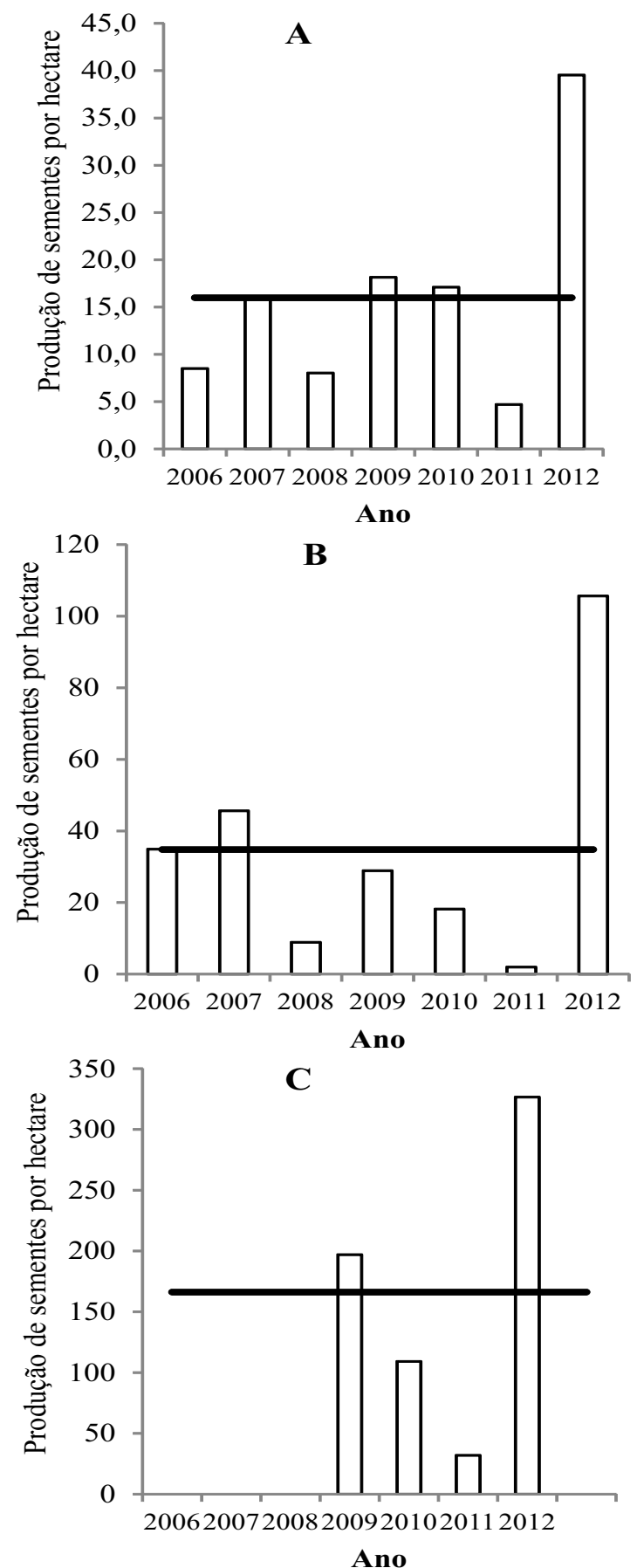

Figura 2 - Variações anuais na produção total de sementes por hectare de castanhais nativos em Roraima: São João da Baliza (A), Caracaraí-Itã (B) e CaracaraíCujubim (C).

Figure 2 - Total seed production annual changes (ha) of brazil nut groves in Roraima: São João da Baliza (A), Caracaraí-itã (B) and Caracaraí-Cujubim (C).
Observou-se que a maior parte da produção se concentrava em poucos indivíduos. Em SJB, as árvores consideradas de alta produção, representadas por cinco indivíduos (14,7\% do total de plantas), foram responsáveis por $41 \%$ da produção total. Em ITA, 54 indivíduos (22,7\% do número de árvores) produziram 74,6\% do total, enquanto em CUJ 17 árvores (15\% do total) tiveram produção de 43,4\%.

Ao analisar as variações anuais nas árvores de maior produção, observou-se diferença no padrão e forte irregularidade, sendo, de forma geral, um ano de alta produção precedido por anos de baixa (Figura 3).

Apenas um indivíduo em SJB não apresentou pico de produção em 2012 (Figura 3 A). No entanto, não foi observado um padrão comum, pois os indivíduos produziram mais ou menos em anos diferentes.

Em ITA, o padrão de produção predominante foi o bianual, com as árvores apresentando picos de produção a cada dois anos (Figura 3B); porém, foram observadas árvores com picos de produção a cada quatro, cinco e seis anos (Figura 3D-F, respectivamente). Em CUJ, foram identificados dois padrões, com árvores apresentando picos de produção a cada dois e três anos (Figura 3C).

\section{DISCUSSÃO}

\subsection{Variações na produção entre populações e indivíduos}

Na comparação com outras regiões da Amazônia, a população CUJ pode ser considerada bastante produtiva, pois a produção de frutos obtida foi próxima à relatada por Zuidema (2003), nas regiões de Madre de Dios, Pando e Beni, na Bolívia, onde se encontraram valores variando de 100 a 184 frutos por árvore. A produção de castanhas, no entanto, foi superior à relatada por Muller (1995 apud PIMENTEL et al., 2007), em clones de castanheiras em plantios comerciais, com produções médias esperadas de até $12,5 \mathrm{~kg}$ de sementes por árvore. Em SJB e ITA, a produção média de frutos foi inferior à observada por Kainer et al. (2007) em cinco anos de monitoramento da Reserva Extrativista Chico Mendes, no Leste do Estado do Acre, com média de 66,2 frutos ( $\mathrm{N}=140$ árvores), porém próximas à observada por Viana et al. (1998), RESEX Cachoeira em Xapuri (AC), no período de 1992-1993, com média de 18 frutos $(\mathrm{N}=141)$.

Revista Árvore, Viçosa-MG, v.38, n.1, p.133-144, 2014 
A semelhança no padrão de produção entre populações relativamente distantes (50 km a $150 \mathrm{~km}$ ) e as diferenças de produção entre os anos sugerem que os fatores de produção globais, como as variações climáticas, podem ter maior efeito sobre o padrão de produção do que fatores locais, como solo, relevo, densidade, estrutura das populações, composição florística, abundância de polinizadores etc. Esses fatores parecem ter maior efeito sobre a magnitude da produção, pois, em média, foram observadas diferenças de até $150 \mathrm{~kg} \mathrm{ha}^{-1}$ entre a região de São João da Baliza e Cujubim, com uma densidade de 2,5 e 5,9 árvores adultas por hectare, respectivamente.
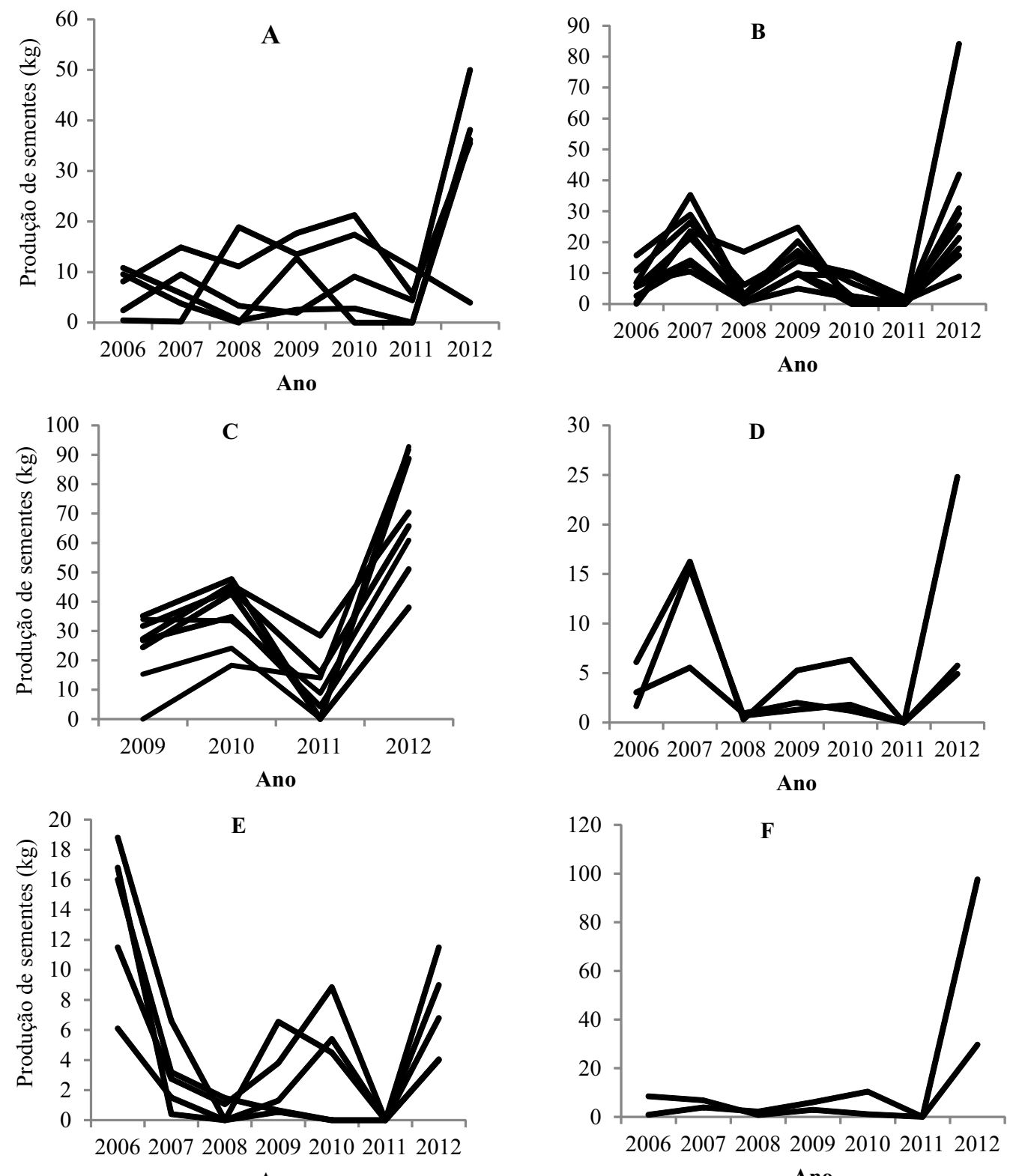

Ano

Ano

Figura 3 - Padrão de produção de sementes observado em São João da Baliza (A), Caracaraí (ITA) - bianual (B), Caracaraí (COJ) - bianual (C) e Caracaraí (ITA) com picos de produção de 4 (D), 5 (E) e 6 (F) anos.

Figure 3 - Seed production pattern observed in São João da Baliza (A), Caracaraí (ITA) - biennial (B), Caracaraí (COJ) - biennial (C) e Caracaraí (ITA) with production peaks of 4 (D) 5 (E) and 6 (F) years. 
Kainer et al. (2007) observaram redução significativa na produção de árvores de castanheira-do-brasil com a redução da precipitação em anos de seca prolongada, enquanto Zuidema (2003) observaram redução em anos de "El Niño".

Ivanov (2011), ao estudar o efeito de diversas variáveis dendrométricas, anatômicas e de clima e solo sobre a produção nesses castanhais, concluiu que a precipitação do mês de setembro apresentou correlação positiva com a produção. Esse mês é caracterizado por diminuição sensível na precipitação no Estado de Roraima, caracterizando-se como um período de transição entre as épocas chuvosa e seca. Como setembro é o mês que antecede a emissão dos botões florais (TONINI, 2011), maior precipitação em setembro pode estimular a produção de botões florais.

A maior produtividade do castanhal CUJ, em relação aos demais, ainda não pode ser explicada de forma plenamente satisfatória, sendo necessária a realização de estudos que busquem aprofundar o conhecimento sobre os sítios em questão. Porém, pode ser explicada, pelo menos parcialmente, a partir de estudos já realizados na região por Tonini (2008) e Ivanov (2011), além de estar relacionada à superioridade genética das plantas ocorrentes.

A análise da estrutura das populações indicou que as árvores localizadas em CUJ são mais grossas (maior média de DAP) e, proporcionalmente, que o número de indivíduos nas classes de maior produção (DAP> $100 \mathrm{~cm}$ ) também é superior nessa região (TONINI et al., 2008). A maior produção de frutos em árvores maiores ocorre devido, provavelmente, à maior capacidade de capturar a luz e à maior biomassa radicular, o que pode diminuir o estresse por umidade durante anos de pouca chuva (SNOOK et al., 2005).

A fragmentação da floresta que ocorreu em ITA e SJB é outro fator que pode ter influenciado na menor produção individual observada em comparação com a CUJ. A castanheira-do-brasil é uma espécie de fecundação cruzada, cuja produção de frutos depende da ação de polinizadores, como as abelhas Euglossine, e, portanto, qualquer desequilíbrio que afete a população de polinizadores irá refletir diretamente sobre a produção de frutos (WHITMORE, 1991; MAUÉS, 2002).

Ivanov (2011) observou que o tipo de solo interagindo com a precipitação apresentou grande influência na produção de sementes nesses locais, constatando maior predominância de solo franco argiloso arenoso profundo em CUJ e solo argiloso raso, onde se obtiveram as piores produções em ITA.

Em comparação com os valores do coeficiente de variação observados por Kainer et al. (2007) na RESEX Chico Mendes $\left(\mathrm{CV}_{\mathrm{p}}=0,19 ; \mathrm{CV}_{\mathrm{i}}=0,88\right)$, no Acre, as variações anuais de produção observadas em Roraima foram bem maiores, o que indica variações distintas entre populações relativamente próximas e em diferentes regiões da Amazônia.

Segundo Zuidema (2003), em populações naturais de castanheiras a variação anual na produção de frutos em uma população é inferior à individual, o que se mostrou válido apenas em São João da Baliza. Neste estudo, a variação anual populacional $\left(\mathrm{CV}_{\mathrm{p}}\right)$ na produção de frutos diferiu em relação ao local, podendo ser considerada baixa em São João da Baliza e muito alta na região de Itã.

A menor densidade de castanheiras na área estudada por Kainer et al. (2007), com 1,35 árvore ha-1, pode ter contribuído para as diferenças observadas, uma vez que os valores obtidos por esses autores estão mais próximos aos de áreas menos densas, em Roraima. Outros fatores podem ter sido o tempo e o período de monitoramento, pois no intervalo observado por Kainer et al. (2007) não foram observados anos de superprodução ou isso não ocorre na região.

O percentual de árvores que não produziram durante o período foi pequeno, estando próximo dos observados por Zuidema e Boot (2002) e Kainer et al. (2007), com $7,5 \%$ e $6,4 \%$, em três e cinco anos de monitoramento, respectivamente.

Segundo Zuidema (2003), no Norte da Bolívia também foram identificados indivíduos adultos não reprodutivos (DAP entre 50 e $120 \mathrm{~cm}$ ). O percentual observado por esses autores (16\%) foi superior aos verificados em Roraima e no Acre, porém o número de árvores e a área pesquisada naquele estudo foram bastante superiores, com 900 árvores e 800 ha, respectivamente.

\subsection{Os castanhais apresentam anos de picos de produção (mast years?)}

Os valores de $\mathrm{CV}_{\mathrm{p}}$ observados em Roraima e o fato de a dispersão de frutos em São João da Baliza ser sincrônica (TONINI, 2011) podem caracterizar a espécie como apresentando anos de pico de produção (mast years), padrão de produção que, segundo Kelly

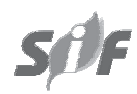

Revista Árvore, Viçosa-MG, v.38, n.1, p.133-144, 2014 
e Sork (2002), é caracterizado por três fatores: variabilidade, sincronia e periodicidade. Para Kellly (1994), um dos critérios que caracterizam esse tipo de frutificação é um $\mathrm{CV}_{\mathrm{p}}$ maior do que 1.

Corroboram esse resultado o fato de que nos trópicos as espécies com registro desse tipo de comportamento estão relacionadas às famílias Lecythidaceae e Dipterocarpaceas (van SCHAIK et al., 1993), e, independentemente do local, houve tendência de maior produção de sementes em um mesmo ano (Figuras 1 e 4), o que para Fenner (1998) também caracteriza o comportamento "masting”.

Os valores de $\mathrm{CV}_{\mathrm{p}}$ observados em ITA e CUJ ( $\mathrm{CV}_{\mathrm{p}}$ $=2,44 ; \mathrm{CV}_{\mathrm{p}}=1,43$ ) estão acima do valor médio de 1,1 obtido por Wright et al. (2005) em 108 espécies da Ilha de Barro Colorado, Panamá, em um período de observação de até 15 anos.

Zuidema (2003) e Kainer et al. (2007) não consideraram excepcionais as variações anuais na produção de frutos das castanheiras, em comparação com outras espécies tropicais; porém, afirmaram que, em períodos maiores de monitoramento, poderiam ser detectadas maiores variações.

No estudo de Kainer et al. (2007), apenas um ano diferenciou significativamente da média, produzindo metade dos frutos na comparação com os demais, o que corrobora as observações dos extrativistas da região, que afirmaram que nesse local as produções são mais ou menos contínuas entre os anos.

Nas áreas estudadas em Roraima, foram observadas grandes variações, com anos produzindo muito abaixo ou muito acima da média, o que também corroborou as observações dos extrativistas locais ao afirmarem que existem anos de grande produção que ocorrem normalmente a cada quatro anos, o que pode ter sido detectado neste estudo.

Várias hipóteses têm sido propostas para explicar as vantagens seletivas do comportamento "masting”, como a eficiência de polinização, a saciedade dos predadores, a polinização animal, a dispersão animal e a predição ambiental (SHIBATA et al., 2002; YASAKA et al., 2003). A hipótese da saciedade do predador tem sido aplicada a espécies predadas por especialistas (SHIBATA et al., 2002) e parece ser a mais provável para explicar os padrões observados neste estudo.
Segundo Yasaka et al. (2003), três condições devem suportar a hipótese da saciedade do predador: a produção de semente deve ser alta o suficiente para saciar os predadores e assegurar a sobrevivência de algumas plântulas; deve existir intervalo mínimo entre os anos de grande produção que resulte em decréscimo na população de predadores durante esses anos; e deve haver sincronia na produção de sementes entre indivíduos na mesma população.

Essas condições têm sido confirmadas por estudos recentes com a espécie. Wadt et al. (2008), ao estudarem a densidade de plântulas e mudas de castanheirado-brasil em diferentes locais e intensidades de coleta, observaram que a maior abundância de frutos no solo associada à menor taxa de coleta resultou em menor porcentagem de frutos abertos pelas cutias (Dasyprocta leporina, Linnaeus, 1758).

Jorge e Peres (2005) observaram que a abundância local de árvores de castanha-do-brasil resulta em diferenças na densidade e na área de vidas das cutias, e em anos de baixa produção de frutos ocorrem decréscimos populacionais, que podem se estender pelos anos seguintes. Tonini (2011), ao estudar a fenologia da espécie em Roraima, identificou um padrão sincrônico de frutificação e dispersão dos frutos.

Segundo van Schaik et al. (1993), o padrão “masting” ocorre em espécies com sementes grandes protegidas apenas mecanicamente, ou seja, sem proteções químicas contra predadores, como é o caso da castanheira-do-brasil. Em plantas que habitam solos pobres em nutrientes (como é o caso da maioria dos solos amazônicos), a produção anual de frutos e sementes não deve permitir grande crescimento populacional de predadores, sendo vantajoso armazenar carboidratos em uma forma inacessível aos predadores, com maiores produções de sementes em longos intervalos.

Portanto, esse resultado enfatiza a necessidade de realização de estudos de longo prazo, pois espécies de comportamento “masting” são particularmente sensíveis às mudanças climáticas que podem alterar a frequência na produção de frutos, com consequências ainda desconhecidas para a fauna e as milhares de comunidades dependentes do extrativismo na região. 


\subsection{Variações temporais na produção de frutos}

Fortes variações temporais na produção de frutos são comuns em espécies tropicais (WRIGHT et al., 2005) e já eram esperadas para as populações em estudo. No entanto, essas variações se mostraram mais extremas para alguns indivíduos dentro de uma mesma população.

As causas para essas variações envolvem diferentes fatores, como a necessidade de acumular reservas entre grandes produções (FENNER, 1998), e a competição por espaço na copa, uma vez que as flores e os frutos das castanheiras estão presentes na parte final dos galhos e favorecem a regeneração da espécie, pois em anos de grande produção os predadores consomem menor proporção do total de sementes produzidas (ZUIDEMA, 2003).

A concentração da produção total em poucos indivíduos no castanhal parece ser característica comum de várias populações e foi observada por Zuidema (2003) na Bolívia e por Kainer et al. (2007) na RESEX Chico Mendes, no Acre, onde essa concentração foi ainda maior, com 25\% das árvores produzindo $72 \%$ da produção total.

Corroborando os resultados obtidos por Kainer et al. (2007), o padrão predominante observado em Roraima não foi o bianual, com árvores produzindo abundantemente durante um ano, seguido de outro de baixa produção, o que foi citado por Zuidema (2003) como o que predomina na Bolívia. Esse padrão foi observado somente em $38,3 \%$ das árvores avaliadas em Roraima.

\section{CONCLUSÕES}

1) A produção de frutos e sementes varia entre os anos nas populações e entre indivíduos em uma mesma população.

2) No ano de maior produção, um castanhal chega a produzir 52 vezes mais do que em anos de baixa.

3) Nos locais estudados, a produção total concentrou-se em pequeno número de indivíduos, parecendo ser o padrão predominante em castanhais nativos da Amazônia.

4) Observou-se um ano de pico de produção (mast year), diferenciando-se do padrão verificado no Acre e na Bolívia.

\section{AGRADECIMENTOS}

Aos Sr. José Lopes Primo e José Fermino Farias e à Madeireira Vale Verde Ltda., pelo apoio na cessão e logística para a coleta dos dados; aos colegas da Embrapa Roraima Adebaldo Sampaio Teles, José de Anchieta Moreira da Costa e Taiguara dos Santos Pereira, pelo esmero na coleta dos dados; e ao CNPq, à Embrapa Roraima e à equipe do Projeto Kamukaia, pelo apoio financeiro.

\section{REFERÊNCIAS}

BOOT, R. G. A. Extraction of nom-timber forest products from tropical rain forest. Does diversity come at a price? Netherlands Journal of Agricultural Science, v.45, p.439-450, 1997.

FENNER, M. The phenology of growth and reproduction in plants. Perpectives in Plant Ecology Evolution and Systematic, v.1, n.1, p.78-91, 1998.

FUNDAÇÃO DO MEIO AMBIENTE CIÊNCIA E TECNOLOGIA DO ESTADO DE RORAIMA FEMACT. O Brasil do hemisfério norte: diagnóstico científico e tecnológico para o desenvolvimento. Boa Vista: Ambtec, 1993. 512p.

IVANOV, G. B. Influência de variáveis dendrométricas, anatômicas e ambientais na produção de frutos e sementes de Bertholletia excelsa H.B.K. 2011. 96f. Dissertação (Mestrado em Ciências Florestais) - Universidade Federal de Santa Maria, Santa Maria, 2011.

JORGE, M. S. P.; PERES, C. A. Population density and home range size of Red-Rumped Agoutis (Dasyprocta leporine) with and outside a natural brazil nut stand in Southeastern Amazonia. Biotropica, v.37, n.2, p.317-321, 2005.

KAINER, K. A.; WADT, L. H. O.; STAUDHAMMER, C. L. Explaining variation in Brazil nut fruit production. Forest Ecology and Management, v. 250, p. 244-255, 2007.

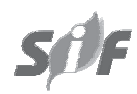

Revista Árvore, Viçosa-MG, v.38, n.1, p.133-144, 2014 
KELLY, D. The evolutionary ecology of mast seeding. Trends in Ecology and Evolution, v.9, p.465-470, 1994.

KELLY, D.; SORK, V. Mast seedling perennial plants: why, how, where? Annual Review of Ecological Systems, v.33, p.427-447, 2002.

MAUÉS, M. M. Reproductive phenology and pollination of the brazil nut tree (Bertholletia excelsa Humb.\& Bonpl.) in eastern Amazônia. In: KEVAN, P.; IMPERATRIZ, F. (Ed.)

Pollinating bees-the conservation link between agriculture and nature. Brasília: Ministério do Meio Ambiente, 2002. p.245-254.

PHILLIPS, O. The potential for harvesting fruits in tropical rainforests: New data from Amazonian Peru. Biodiversity and Conservation, v.2, n.1, p.18-38, 1993.

PERES, C. A. et al. Demographic threats to the sustainability of Brazil nut exploitation. Science, v. 302, p.2112-2114, 2003.

PIMENTEL, L. D. et al. Estimativa de viabilidade econômica no cultivo da castanha- do- brasil. Informações Econômicas, v.37, n.6, p.26-36, 2007.

SALOMÃO, R. P. Densidade, estrutura e distribuição espacial da castanheira-do-brasil (Bertholletia excelsa H \& B) em uma floresta tropical de platô na Amazônia Setentrional.

Boletim do Museu Paraense Emilio Goeldi. Série botânica, v.4, n.1, p.11-25, 2009.

SECRETARIA DE ESTADO DO PLANEJAMENTO E DESENVOLVIMENTO DE RORAIMA - SEPLAN. Informações Socioeconômicas do Município de São João da BalizaRR, 2010. Boa Vista: CGEES/SEPLAN - RR, 2010. 68p.

SHIBATA, M. et al. Synchronized annual seed production by 16 principal tree species in a temperate deciduous Forest, Japan. Ecology, v.83, n.6, p.1727-1742, 2002.
SNOOK, L. K.; CÁMARA-CABRALES, L.; KELTY, M. J. Six years of fruit production mahogany trees (Swietenia macrophylla King): patterns of variation and implications for sustainability. Forest Ecology and Management, v.206, p.221-235, 2005.

SIEGEL, S.; CASTELLAN, N. J. Nonparametric statistics for the behavioral sciences. New York: McGraw-Hill, 1988. 200p.

TONINI, H.; COSTA, P.; KAMINSKI, P. E. Estrutura e produção de duas populações nativas de castanheira-do-brasil em Roraima. Floresta, v.38, n.3, p.445-457, 2008.

TONINI, H. Fenologia da castanheira-do-brasil (Bertholletia excelsa Humb.\&Bonpl., Lecythidaceae) no sul do estado de Roraima. Cerne, v.17, n.1, p.123-131, 2011.

VIANA, V. M. et al. Ecologia e manejo de populações de castanhado-Pará em reservas extrativistas, Xapuri, Estado do Acre. In: GASCON, C.; MOUTINHO, P. (Ed.). Floresta Amazônica: dinâmica, regeneração e manejo. Manaus: INPA, 1998. p.373-390.

van SCHAIK, C. P.; TERBORGH, J. W.; WRIGHT, S. J. The phenology of tropical forests: Adaptative significance and consequences for primary consumers. Annuall Review of Ecological Systems, v.24, p.353-377, 1993.

ZUIDEMA, P. A.; BOOT, R. G. A. Demography of the Brazil nut tree in the Bolivian Amazon: impact of seed extraction on recruitment and population dynamics. Journal of Tropical Ecology, v.18, n.1, p.1-31, 2002.

ZUIDEMA, P. A. Demography and management of the Brazil nut tree (Bertholletia excelsa). Riberalta, Bolivia: PROMAB, 2003. 111p. (Scientific Series, 6)

YASAKA, M.; TERAZAWA, K.; KOYAMA, H. K. H Masting behavior of Fagus crenata in northern Japan: spatial synchrony and pre-dispersal seed predation. Forest Ecology and Management, v.184, p.277-284, 2003. 
WADT, L. H. O.; KAINER, K. A.; GOMES-SILVA, D. A. P. Population structure and nut yield of a Bertholletia excelsa stand in Southwestern

Amazonia. Forest Ecology and

Management, v.211, p.371-384, 2005.

WADT, L. H. O. et al. Sustainable forest use in Brazilian extractive reserves: natural regeneration of brazil nut in exploited populations. Biological Conservation, v.141, p.332-346, 2008.
WHITMORE, T. C. Tropical rain forest dynamics and its implications for management. In: GOMEZPOMPA, A.; WHITMORE, T. C.; DADLEY, M.

(Ed.).Rain forest regeneration and management. New Jersey: United Nations Educacional Scientific and Cultural Organization, 1991. p.67-86.

WRIGHT, S.J. et al. Annual and spatial variation in seedfall and seedling recruitment in a neotropical forest. Ecology, v.86, n.4, p.848860, 2005. 
